\title{
Enhancing Power Saving Mechanisms for Ad hoc Networks
}

\author{
Wafa Akkari and Abdelfettah Belghith
}

\author{
Wafa Akkari \\ HANA Research Group, ENSI, University of Manouba, Tunisia 2010, e-mail: \\ wefa.akkari@gmail.com \\ Abdelfettah Belghith \\ HANA Research Group, ENSI, University of Manouba, Tunisia 2010, e-mail: abdelfat- \\ tah.belghith@ensi.rnu.tn
}

\begin{abstract}
According to PSM, each station must announce traffic before its transmission. PSM requires for this purpose a rather large handshaking period to complete the required announcements. This functional behavior puts a heavy constraint on the size of the announcement period and consequently on throughput, delay and more importantly on power consumption. This paper proposes an optimization of some functional properties of PSM in order to improve its performances. Our proposed UTA-PSM (Unicast Topology Aware Power Saving Mechanism) aims to reduce the number of exchanged announcement frames in order to reduce the announcement period. Conducted simulations show that UTA-PSM outperforms superbly PSM and power saving mechanisms such as the DCS-ATIM that dynamically adjusts the handshaking period according to the traffic load. We show that UTA-PSM delivers more throughput with a smaller average delay yet yielding a much better power saving and robustness.
\end{abstract}

\section{Introduction}

As wireless networks become an integral component of the modern communication infrastructure, energy efficiency becomes an important challenge due to the limited battery life of mobile terminals. Two different operational modes are defined in IEEE802.11 [2], the dominating WLAN technology today: the infrastructure network in which a specific central entity manages communications between stations, and the ad hoc network where spontaneous mobile nodes (we shall use interchangeably the word node or station) communicate with each other over multiple wireless hops $[2,8]$. As each mobile node in an ad hoc network performs routing functionalities to assist other node communications, even one or few mobile hosts' energy exhaustion might cause the disruption of the entire network [16]. Therefore, there has been an increased interest in energy efficient communication protocols for the ad hoc networking environment $[6,25,13,22,7]$ and numerous energy efficient MAC 
protocols have being proposed $[5,10,24,3,4]$. These protocols are virtually all based on turning off the nodes' radio transceivers (hence putting the transceiver in the so called sleep or Doze state) to reduce energy consumption whenever they are not involved in communication. Neither a transmission nor a reception is allowed when a node is in this inactive state thus resulting in little energy consumption. The IEEE 802.11 DCF (Distributed Coordination Function) standard [2] also incorporates such a power saving mechanism (PSM) that uses Awake and Doze states. In 802.11 PSM, time is divided into specific periods called Beacon intervals and each node tries to synchronize with its neighbors to ensure that all nodes wake up at the same time. Any node can announce its pending data information during the subperiod called the ATIM window using the ATIM (Announcement Traffic Indication Map) frames which must each be acknowledged by an ATIM-ACK frame. During the period following ATIM window (we here denote this period as the BeyondATIM window or period), nodes having sent an ATIM or an ATIM-ACK frame must remain in an Awake state and perform data communication based on Carrier Sense Multiple Access with Collision Avoidance (CSMA/CA) mechanism, whereas all other nodes switch to Doze state. It should be stressed here that the number of ATIM frames may be very important as each station needs to announce its pending traffic. The required ATIM window may take a huge fraction of the Beacon period leaving insufficient Beyond-ATIM period to transmit the announced data frames. A compromise between the length of the ATIM period and the Beyond-ATIM period is surely needed and several research works have tackled such an issue. The central question here is how can we lessen this ATIM window to its minimum, yet allowing the maximum of announcements and data traffic exchange. To this end, we propose a new protocol coined UTA-PSM (Unicast Topology Aware-PSM) that thrives to decrease the ATIM window period. This is accomplished by making each node aware of the already known nodes that will stay Awake during the following Beyond-ATIM period. Indeed all nodes are readily Awake during the ATIM window and hence they are inspecting all transmitted ATIM and ATIM-ACK frames that are transmitted within their respective vicinities. These control frames readily provide the needed information about which nodes have already agreed to stay Awake for the data transmission phase. UTA-PSM simply allows a node to maintain such an information and abandon its ATIM transmission if the destined node of the ATIM is already sensed to stay anyway active. In this way and as we shall later show, we can significantly reduce the handshaking control traffic and consequently a substantially smaller ATIM period is needed. A smaller ATIM window not only implies that more time is left for the data exchange phase but more importantly that more announcements could be made which clearly amounts to a better throughput. To our best of knowledge no one has used this context aware handshaking in ad hoc networks. We shall show that this will provide a better throughput and smaller delays, yet amounts to much better energy saving than some of the most reputed protocols.

The rest of the paper is organized as follows. In section (2), we present a quick survey on recent research related proposals. We purposely put the accent on proposed energy saving protocols that dynamically adjust the ATIM period as a function of the traffic load. We devote section ( 3 ) to introduce and explain our proposed 
UTA-PSM. Section ( 4) explains our experimental set up and results. Finally, section (5) presents some concluding remarks and future directions.

\section{Related work}

Power save protocols take a variety of forms. IEEE 802.11 PSM [2] attempts to conserve energy on idle nodes by powering off their wireless interfaces for a specific period of time. Nodes are assumed to be synchronized and should get Awake at the beginning of each Beacon interval. The synchronization is established by exchanging specific management frame called Beacon according to the TSF (Timing Synchronization Function) function [2]. At every Beacon interval, each node must even send a Beacon or receive at least one. TSF uses timestamped Beacon transmitted to synchronize clocks among nodes.

After waking up at the beginning of the Beacon interval, each node stays on for a period of time called the ATIM window (Ad hoc Traffic Indication Message window). During the ATIM window, since all nodes are guaranteed to be listening, queued data frames are advertised by sending a Unicast ATIM frame. Upon reception of an ATIM frame, a station replies by sending an ATIM-ACK. The transmission of the ATIM frame is performed using the CSMA/CA mechanism specified in IEEE 802.11. No data is sent during the ATIM window. Upon the termination of the ATIM window, any station that has transmitted ATIM frames (of which at least one is acknowledged) or an ATIM-ACK remains active during the entire current period and attempts to send its announced data frames before the next Beacon interval. Any node that has not transmitted an ATIM or an ATIM-ACK frame enters the Doze state at the end of the ATIM window until the start of the next Beacon interval. Figure 1 above illustrates a typical data transfer in a three nodes ad hoc network. Station A sends an ATIM to station B which responds by sending an ATIM-ACK during the same period. Both stations A and B must remain Awake for the whole

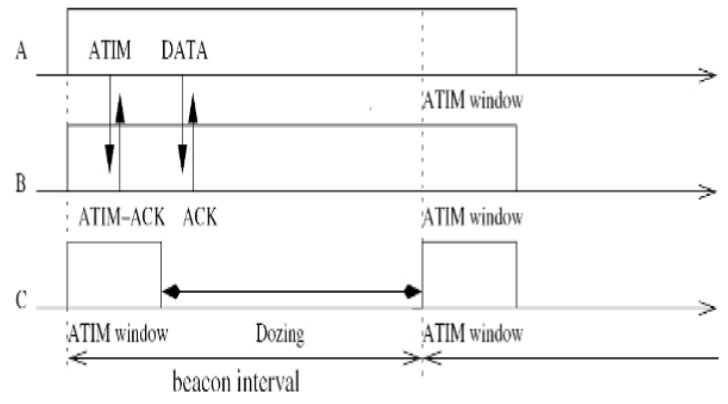

Fig. 1 Power management in an ad hoc network using PSM. 
Beacon interval. Station C can switch to sleep state at the end of the ATIM window since it has not sent or received any ATIM frame. This means that, in a dense network, with many traffic flows, we need to have a large announcement period (ATIM window) so that all sources can send their ATIM frames and receive the correspondent ATIM-ACK. However, for a predetermined Beacon interval period, increasing the ATIM window period leads to the decrease of the Beyond-ATIM window period and consequently the time devoted to data transmission is reduced. This way of functioning can degrade the throughput and increase the delay.

In [9], the authors proposed an adaptive scheme to reduce power consumption and forwarding delay in an ad hoc network. APSM (Adaptive Power Saving Mode) allows stations to have multiple Awake instants during the same Beacon interval. The Awake instants are decided by the station receiving the ATIM during the ATIM window and includes them into the ATIM-ACK. Both stations know when to get Awake to handle their data traffic. The number of the Awake instants can be adjusted using traffic information. The problem with APSM is that having many Doze-to-Awake transitions may consume an important amount of energy. In fact, the transition from the Doze state to the Awake state consumes more energy than transmitting [20, 23].

In [4], the others argue that having a fixed ATIM window the same for all nodes may not be efficient. The size of this window can be too small to receive and send all announcements or too large for the Beacon interval to be able to deliver all data traffic. They presented DPSM (Dynamic Power Saving Mechanism) as an improvement to PSM that allows to a node to dynamically and locally choose a suitable size for its own ATIM window. In particular, they showed that the ATIM window depends on both the traffic load and the number of nodes in the network. However, the procedure of dynamically choosing the ATIM window size works perfectly only in a fully connected network (i.e., just one cell) and fails to be efficient for multi-hop networks.

IPSM (Improved Power Saving Mechanism) first presented in [12] and then in [11] stipulates that the ATIM window ends when the channel is idle for a specified amount of time. However, IPSM only works in single-hop networks since it relies on a node and all its neighbors having the same consistent view of channel activity. Some nodes (all the nodes of the network in some cases) can increase their ATIM window period to ATIMmax for the wrong reasons: the channel can be idle because of other's data transmission or simply interference.

In [18], the authors propose the use of carrier sensing to dynamically adjust the size of the advertisement windows. DCS-ATIM (Dynamic Carrier Sense-ATIM) allows nodes to have different values of ATIM window depending on the amount of traffic that needs to be advertised in the current window. Carrier sensing is used as an energy efficient method to let neighbors know if a node intends to advertise any packets in the upcoming window. Carrier sensing is also used as a mechanism for nodes to keep track of whether their neighbors have already stopped listening for advertisements and possibly returned to sleep. The authors show that DCS-ATIM can significantly reduce the energy consumption of 802.11 PSM with a slight increase in latency. The authors stipulated to the contrary of IPSM, that DCS-ATIM stays 
efficient even in multi-hop networks. We shall show in the following sections that DCS-ATIM is only effective when traffic load is extremely light. For other traffic conditions even very moderate, DCS-ATIM delivers worse than PSM due to time it takes for sensing.

In [10], they proposed an extensible on demand power management scheme that adapts to traffic load. Nodes maintain timers to determine power management transitions. They proposed to monitor routing control messages and data transmissions to set and update the timers. Nodes that are not involved in data delivery may then enter the Doze state.

SPAN [5] elects a group of nodes, called coordinators to be responsible for forwarding the traffic to active connections. Thus, the coordinators must stay Awake however, the non-coordinator nodes follow the power saving mechanism in IEEE 802.11 PSM. The coordinators are periodically changed to face the problem of mobility and battery depletion. SPAN defines a new advertisement traffic window following the ATIM window. During this window, the announced packets (during the ATIM window) can be transmitted in addition to the traffic among coordinators. After this window, only traffic among coordinators can be transmitted. It is worth here mentioning that the clustering phase needed to select coordinators and the time needed to maintain the clustered topology would greatly affect the performance of SPAN. The authors just assumed that this is done with no traffic taken into account in their evaluations.

\section{Proposed UTA-PSM scheme}

We now present our proposed scheme, UTA-PSM (Unicast Topology Aware Power Saving Mechanism). The main difference between UTA-PSM and PSM is that nodes can transmit data frames even if they didn't sent any announcement frame during ATIM window. Each station maintains an active neighbors table (ANT) that contains the identities of the neighbors learned to stay Awake during the Beyond-ATIM window of the current Beacon period. ANT is initialized to be empty at the start of each Beacon period. During the ATIM period, whenever a station hears an announcement frame (ATIM or ATIM-ACK) it adds, to its ANT, the identity of the transmitting node if it is not already here. A station refrains to transmit an ATIM frame to any station indicated in its ANT since anyway that station will surely be Awake. The ATIM and the ATIM-ACK transmitting nodes are considered as Awake neighbors for the entire current Beacon interval. However, we recall that the ATIM frame contains both the source and the destination identities but the ATIM-ACK contains only the ATIM-ACK receiving station address [2]. Consequently, we propose to modify the ATIM-ACK frame to also include the ATIM-ACK source address. This simple modification allows nodes to be aware of the state of all their neighbors being in Doze or Awake state. Being Awake results from the transmission of at least one ATIM or ATIM-ACK frame. A station receiving an ATIM frame destined to itself must, after performing the required updating to its ANT, send an 
ATIM-ACK frame and consequently stays Awake for the entire Beacon period. A station sends its ATIM if it is destined to a node not yet included in its ANT. Finally, a station goes into the Doze state if it has no data frame to send and it has not acknowledged any ATIM frame.

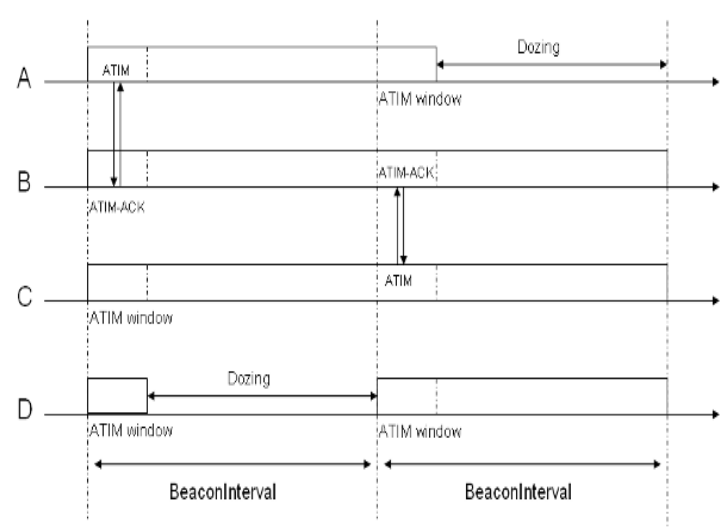

Fig. 2 Power management in an ad hoc network using UTA-PSM.

Using Figure 2, we will explain how UTA-PSM works. All stations are supposed for the sake of simplicity to be in line-of-sight. During the first Beacon interval, stations $\mathrm{A}$ and $\mathrm{C}$ have packets to send to node B. Both A and C prepare an ATIM frame to be sent to B according to the underlying CSMA/CA scheme. In Figure 2, we suppose that A transmits first to B. B, C and D being all neighbors of A will firstly update their ANTs by including A's identity. Then, B directly acknowledges the received ATIM frame by sending an ATIM-ACK frame to A. A, C and D will then update their ANTs by including B. Exactly at the same time, node C aborts its pending transmission to B. Station D however, having no traffic to announce, can switch to the Doze state at the end of the ATIM window. At the end of this Beacon period all ANTs at the different nodes will be flushed. For the second Beacon period, we suppose that node B sends its announcement ATIM frame before D. The rest of the scenario continues in the same way.

UTA-PSM may significantly reduce the number of exchanged announcement frames in comparison to PSM and DCS-ATIM. This will certainly allow to specify a much smaller ATIM window. We can further improve UTA-PSM by making it more sensitive to the traffic load. To carry out this objective, we referred to the work presented in [1]. In this work, the authors investigated in particular enhancement called the Traffic Aware Power Saving Mechanism (TA-PSM) that allows nodes to enter the Doze state in the middle of a Beacon period when they are no more involved in data delivery even if they have already sent an ATIM or an ATIM-ACK. TA-PSM reduces the energy consumption by making PSM more sensitive to the current traffic. First, they added a one bit MoreData field into the frame header to 
indicate to the receiving station that further pending data frames are buffered. If the MoreData bit is set in the received frame, both the transmitting and the receiving stations stay Awake; otherwise, they can switch to the Doze state. Our scheme consists on reducing the energy consumption by making UTA-PSM more sensitive to the traffic load in the same way TA-PSM does. Thus, the same idea is applied on UTA-PSM and all stations having finished their data transmission without having sent an ATIM or an ATIM-ACK may switch to the Doze state to save energy. Stations having sent an ATIM or an ATIM-ACK cannot switch to the Doze state during the Beyond-ATIM period because other stations rely on them.

\section{Simulation results}

We implemented our simulated models using J-Sim [14, 21]. We simulate the proposed UTA-PSM scheme, the PSM scheme, the DCS-ATIM mechanism proposed in [18] and IEEE 802.11 without power saving. For these schemes, we use the following abbreviations:

- WOPSM: This is the IEEE 802.11 protocol with no power saving mechanism.

- PSM: This is the standard IEEE 802.11 protocol with power saving enabled.

- UTA-PSM: Our proposed protocol described in Section 3.

- DCS-ATIM: This is 802.11 PSM with the dynamic ATIM modification for multihop networks described in [18].

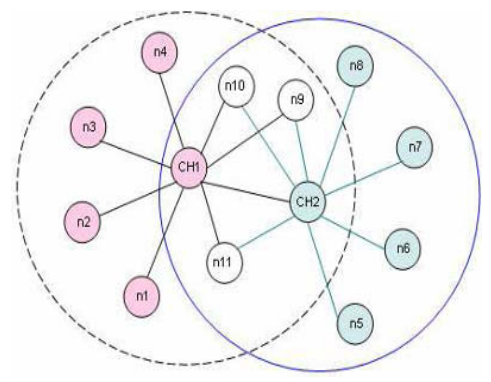

Fig. 3 The network topology.

We use four metrics to evaluate the proposed UTA-PSM and compare its performance with the others:

- Aggregate Throughput: represents the total number of data frames delivered to all destined stations at the end of the simulation. This metric is useful to verify whether power saving mechanisms degrade throughput or not. 
- Delay: represents the average time a data frame spent in the network from its generation at the source station to its delivery to the destination station.

- Power consumed: representing the total energy consumed by all stations during the simulation time.

- Power consumed per delivered data frame: represents the total energy consumed divided by the number of delivered data frames.

Each simulation run is performed for 200 seconds. We used CBR traffic models with different data rates, a fixed data frame size of 512 bytes, a wireless channel bit rate of 2 Mbps. The transmission range is fixed to $240 \mathrm{~m}$. We considered the network example depicted on Figure 3. The data flows are defined as follows: n1CH1-CH2-n7, n2-CH1-CH2-n8, n10-CH1-n4, n11-CH2-n5, n4-CH1-n11, n5-CH2n8, n9-CH1-n3 and $\mathrm{CH} 1-\mathrm{CH} 2$. Stations $\mathrm{CH} 1$ and $\mathrm{CH} 2$ are the clusterheads and the rest of stations of the network are just ad hoc mobile stations. We choose to emulate a clustered network to show best the contribution of UTA-PSM. In fact all the defined flows have to be forwarded through $\mathrm{CH} 1, \mathrm{CH} 2$ or both of them which concentrate traffic through this two particular stations. Having all stations involved in data transmission in a clustered topology is more in favor of UTA-PSM than of DCS-ATIM because the advantage of UTA-PSM is especially shown when the topology of the network presents a centrifugal architecture. We consider a Beacon interval of 0.1 seconds which is the default value specified for PSM in [6]. We assume an initial energy equal to 1000 Watt per station. The energy consumption model used throughout the simulations is defined as follows:

- Energy consumption rate in transmit state equals 0.660 Watt per second.

- Energy consumption rate in receive state equals 0.395 Watt per second.

- Energy consumption rate in idle state equals 0.296 Watt per second.

- Energy consumption rate in Doze state equals 0.0 Watt per second.

We first tested the throughput and power consumption of PSM and UTA-PSM to fix the appropriate ATIM window for each mechanism. The ATIM window attributed to DCS-ATIM is the same value PSM has as indicated in [18]. For these tests, we fixed the traffic load of each flow to $13 \mathrm{pkt} / \mathrm{sec}(52 \mathrm{kbps})$ and we varied the length of the ATIM window from $2 \mathrm{msec}$ to $25 \mathrm{msec}$.

As shown in Figure 4(a), both of the power saving mechanisms naturally show an increase followed by a decrease in throughput as the ATIM window is increased. If the ATIM window is too small, ATIM frames won't be sent so no data frame will be sent. On the other hand, if the ATIM window is too large, there won't be enough time to transmit the announced data frames and hence throughput decreases. More importantly, we observe that UTA-PSM delivers far more aggregate throughput than does PSM since UTA-PSM needs a much shorter ATIM period. On the other hand, Figure 4(b) portrays the total power consumed by the two schemes. It is clear that UTA-PSM consumes far more power since it delivers far more data frames. Recall that the energy consumption rate in transmit state is the highest. These two curves allow us to set the adequate ATIM window corresponding to each of the two schemes. Hereafter, we shall then use these typical values of the ATIM window $15 \mathrm{msec}$ for PSM and 6 msec for UTA-PSM. 


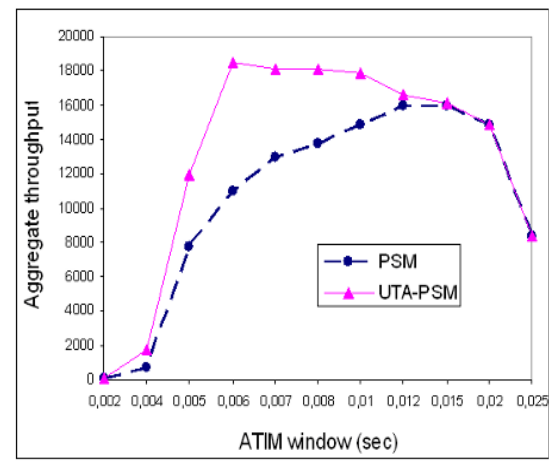

(a)

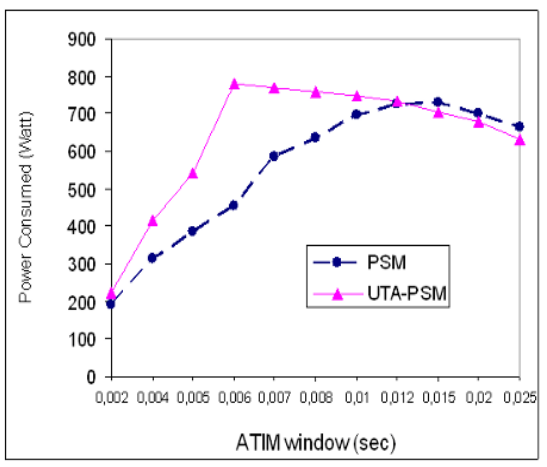

(b)

Fig. 4 (a)(b): Aggregate Throughput and Power consumed vs ATIM window.

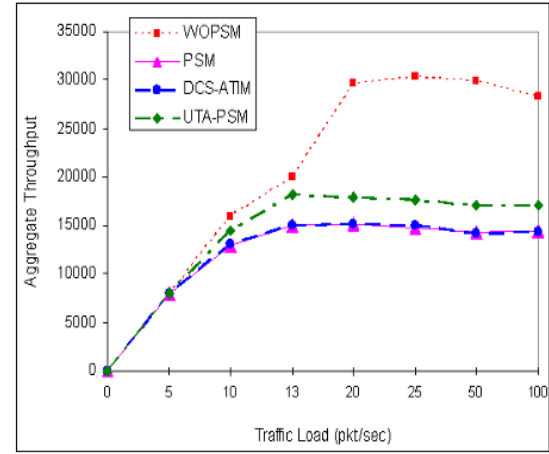

(a)

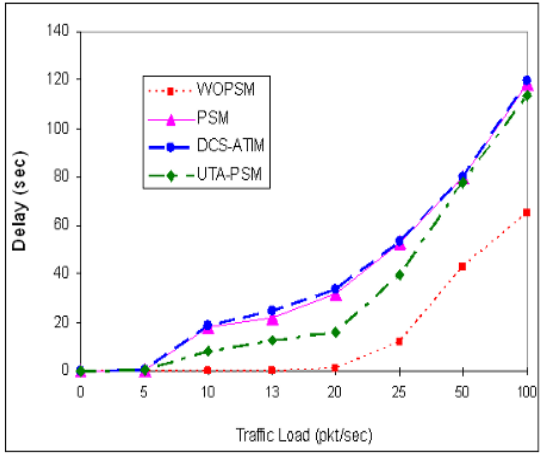

(b)

Fig. 5 (a)(b): Aggregate Throughput and Delay vs Traffic Load.

Now let us consider the same topology (Figure 3) and vary the data rate of all the defined flows from 5pkt/sec to 100pkt/sec. We clearly observe that PSM and DCSATIM yield the same throughput: the corresponding curves are completely superposed in Figure 5(a). However, UTA-PSM performs significantly better than both PSM and DCS-ATIM but not better than WOPSM that offers the highest throughput. This difference between WOPSM and all other protocols is readily justified by the influence of the ATIM window during which no data frame is sent.

Now, let us consider the delay of all received frames. Figure 5(b) portrays the delay and shows that PSM and DCS-ATIM still have almost superposed curves (slightly less for PSM). The difference between the delay for DCS-ATIM and the delay provided by PSM is relatively constant in the range of $800 \mathrm{msec}$ to $1 \mathrm{sec}$. This small increase in DCS-ATIM latency comes from the fact that that packets arriving after the ATIM window is expired will not be announced until the next ATIM 


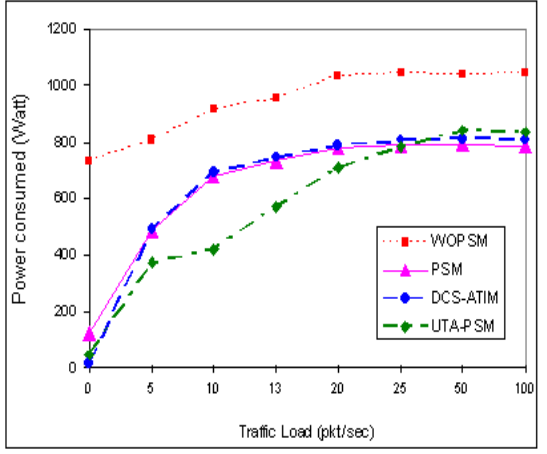

(a)

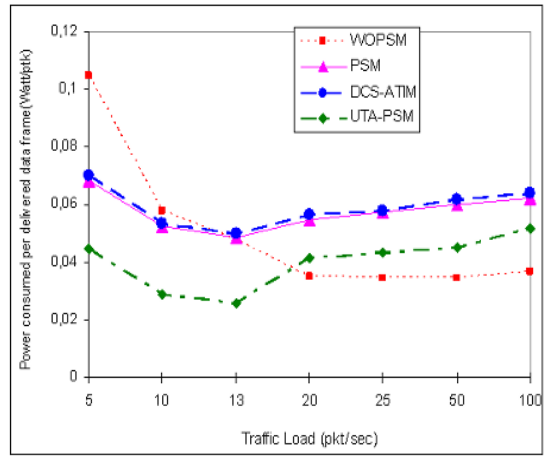

(b)

Fig. 6 (a)(b): Power consumed and Power consumed per delivered data frame vs Traffic Load.

window in the case of DCS-ATIM. Another fact that makes the difference in delay between PSM and DCS-ATIM is the extra carrier sensing period. UTA-PSM, on the other hand, outperforms clearly both DCS-ATIM and PSM. It delivers more throughput and yields less delay.

Now, we focus on UTA-PSM main efficiency as a power saving mechanism. In Figure 6(a) below, we show the total power consumed by all nodes in the network as a function of the traffic load. DCS-ATIM consumed energy is less than that consumed by any of the other schemes for very extremely small traffic loads only. This is indeed due to its dynamic ATIM window which is kept smaller than even that of UTA-PSM. However, DCS-ATIM leverages and from above the energy consumed by PSM when the traffic load gets larger than 5 packets per second per traffic source. In fact, the energy consumption of DCS-ATIM converges from above to that of PSM since it additionally needs the overhead for carrier sensing. However, we observe that UTA-PSM outperforms DCS-ATIM and surely PSM when the traffic load gets larger than 2 packets per second. This is somehow remarkable for back to Figure 5(a) we see that UTA-PSM delivers more throughput also. That is to say: despite the energy consumed by transmitting more packets (recall that transmitting energy consumption rate is the largest), UTA-PSM consumes less energy. To this end, we consider in Figure 6(b) the average power consumed per delivered data frame as a function of the traffic load. This is just the total energy consumed by all nodes divided by the total network throughput. This figure shows that UTA-PSM clearly outperforms PSM and DCS-ATIM which have almost the same power consumed per delivered data frame. At light traffic conditions, where any energy saving mechanism is supposed to save the most, we notice the clear efficiency of UTA-PSM, a substantial gain of more than $40 \%$ over both PSM and DCS-ATIM (at a traffic load of 5 packets per second). As the traffic load increases, the gain decreases a little bit until reaching a limiting value which indicates the gain accomplished by the reduction of the number of announcement frames exchanged during ATIM window. It is 
also worth noticing that at high traffic loads it becomes better not to use any power saving mechanism. In this region we readily see that WOPSM outperforms all the studied schemes and for that matter any power saving mechanism as nodes are kept Awake all the time. Nevertheless, it is interesting to notice that UTA-PSM can save energy where the others fail completely.

\section{Conclusion and future directions}

IEEE 802.11 PSM and the like that base their saving mechanisms on a handshaking period whether this period is predefined and fixed or dynamic do not yield as expected good performances. The crux of this is that the number of announcements should be lowered at its maximum to give more time to data frames and in turn obtain more throughput. This is exactly what our proposed UTA-PSM is about. Lowering the handshaking period is shown to allow more announcements and more throughput. This in turn yielded a much better data frame average delay. Conducted simulations portrayed that UTA-PSM outperforms not only PSM but more importantly schemes such as DCS-ATIM that dynamically adjust their ATIM period. The later has been shown to behave as poorly as PSM since when traffic load is not extremely light the ATIM window gets at least the same value as that used by PSM. Our simulations were based on a network topology that is supposed to be clustered. This indeed let traffic flows trough certain nodes, namely the clusterheads. Other scenarios could use routing algorithms that have a similar effect of concentring traffic through certain nodes such as the OLSR algorithm. Unquestionably more simulations are needed to better ascertain the excellent efficiency of UTAPSM. Furthermore efforts are underway to integrate the DCS-ATIM approach into our UTA-PSM scheme.

\section{References}

1. Abdelfattah Belghith, Wafa Akkari and Jean Marie Bonnin: Traffic Aware Power Saving Protocol in Multi-hop Mobile Ad-hoc networks. JOURNAL OF NETWORKS, Volume 2, Issue 4, Pages 1-13(2007)

2. ANSI/IEEE Standard 802.11: Wireless LAN Medium, Access Control (MAC) and Physical, Layer (PHY)Specifications. (1999)

3. A. Sheth and R. Han: Adaptive power control and selective radio activation for low-power infrastructure-mode 802.11 LANs. Proceedings of 23rd International Conference on Distributed Computing Systems (ICDCSW'03), Providence, Rode Island, USA, 5: 812-817 (2003)

4. B. Awerbuch, D. Holmer and H. Rubens: The pulse protocol: energy efficient infrastructure access. IEEE INFOCOM'04, Hong Kong (2004)

5. B. Chen, K. Jamieson, H. Balakrishman and R.Morris: Span: An energy-efficient coordination algorithm for topology maintenance in ad hoc wireless networks. in MOBICOM 2001 (2001)

6. C.E. Jones, K. Sivalingam, p. Agrawal and J Chen: A survey of energy efficient network protocols for wireless networks. ACM Journal Wireless Networks , 15: 343-358 (2001) 
7. Changsu Suh, Young-Bae Ko and Jai-Hoon Kim: Enhanced Power Saving for IEEE 802.11 WLAN with Dynamic Slot Allocation. LNCS, Vol. 3794, 11: 466-477 (2005)

8. Conference on Mobile Computing and Networking. San Diego, California, USA, September 14-19, (MobiCom 2003)

9. D.Y. Kim and C.H. Choi: Adaptive power management for IEEE 802.11 based ad hoc networks. in World Wireless Congress, San Francisco, USA (2004)

10. E.S. Jung and N.H. Vaidya: An energy efficient MAC protocol for wireless LANs. INFOCOM 2002,New York, USA (2002)

11. Eun-Sun Jung and N. H. Vaidya: Improving IEEE 802.11 Power Saving Mechanism. University of Illinois at Urbana-Champaign, Tech. Rep (2004)

12. Eun-Sun Jung and Nitin H. Vaidya: Improving IEEE 802.11 power saving mechanism. Published online: 4 January 2007, Springer Science+Business Media, LLC (2007)

13. H. Zhu and G. Cao: A power-aware and QoS-aware service model on wireless networks. IEEE INFOCOM'04, Hong Kong (2004)

14. J-Sim simulator: on-line at www.j-sim.org, last visited in 23-05-2005

15. J. So: Design and Evaluation of Multi-channel Multi-hop Wireless Networks. Ph.D. Dissertation, University of Illinois at Urbana Champain (2006)

16. J. Zhu, C. Qiao and X. Wang: A comprehensive minimum energy routing scheme for wireless ad hoc networks. IEEE INFOCOM'04, Hong Kong (2004)

17. L. Huang and T. lai: On the scalability of the IEEE 802.11 Ad HOC Networks. In the proceedings of the ACM MOBIHOC (2002)

18. Matthew J. Miller and N. H. Vaidya: Improving Power Save Protocols Using Carrier Sensing for Dynamic Advertisement Windows. University of Illinois at Urbana-Champaign, Tech. Rep (2005)

19. N. Bulusu, J. Heidemann, and D. Estrin.: GPS-less low cost outdoor localization for very small devices. IEEE Personal Communications Magazine, 5:28-34 (2000)

20. Paul J.M.Havinga and Gerard J.M.Smit: Energy-Efficient TDMA meduim access control protocol scheduling. in Proc.Asian International Mobile Computing Conference AMOC'02 (2000)

21. R. S. Nair: JSIM: A Java-Based Query Driven Simulation and Animation Environment. Masters Thesis (M.S. in CS Degree) (1997)

22. R. Zheng and R. Kravets: On-demand Power Management for Ad Hoc Networks. Proc. of IEEE INFOCOMM 2003, San Francisco (2003)

23. Tajana Simunic, Haris Vikalo, Peter Glynn and Giovanni De Micheli: Energy efficient design of portable wireless system. in International Symposium of Low Power Electronics and Design (ISLPED) (2000)

24. T. Simunic and S. Boyd: Managing power consumption in networks on ships. ACM DATE (2002)

25. T. Zhang, P. Gurung: E. Van Den Berg, S. Madhani and A. Muttreja, Silent networking for energy constrained nodes. in press in Computer Communications, Elsevier (2006) 